\title{
Separable algebras over infinite fields are 2-generated and finitely presented
}

\author{
Marcin Mazur and Bogdan V. Petrenko
}

\begin{abstract}
We prove that every separable algebra over an infinite field $F$ admits a presentation with 2 generators and finitely many relations. In particular, this is true for finite direct sums of matrix algebras over $F$ and for group algebras $F G$, where $G$ is a finite group such that the order of $G$ is invertible in $F$. We illustrate the usefulness of such presentations by using them to find a polynomial criterion to decide when 2 ordered pairs of $2 \times 2$ matrices $(A, B)$ and $\left(A^{\prime}, B^{\prime}\right)$ with entries in a commutative ring $R$ are automorphically conjugate over the matrix algebra $M_{2}(R)$, under an additional assumption that both pairs generate $M_{2}(R)$ as an $R$-algebra.
\end{abstract}

Mathematics Subject Classification (2000). Primary 16H05, 16K20, 16S15, 16R30.

Keywords. Separable algebras, Generators of algebras, Presentations of algebras, Conjugacy invariants.

1. Introduction. A fundamental method to define and study associative algebras, both finite and infinite dimensional, is by means of their presentations. Some presentations of integer matrix algebras have been found in [8, p. 18]; some closely related questions have been addressed in [1] and [10]. An important infinite dimensional example of an algebra defined by a presentation is a quantum plane (see [5, Chapter 4]); it is a quotient of the free polynomial algebra in two variables by a certain principal ideal. The present paper is a step in the study of presentations of separable algebras over fields. For the convenience of the reader we recall the following definition. ${ }^{1}$

${ }^{1}$ This definition is equivalent to one of the usual definitions of separability (see [9, Chapter 10]). 
Definition 1. Let $F$ be a field, and let $\bar{F}$ be an algebraic closure of $F$. A unital associative algebra $A$ over $F$ is called separable if it is finite dimensional as an $F$-vector space, and if the algebra $A \otimes_{F} \bar{F}$ is isomorphic to a finite direct sum of matrix algebras over $\bar{F}$.

The class of separable $F$-algebras is closed under taking quotients and finite direct sums (see [9, Chapter 10]) and it coincides with the class of finite dimensional semisimple $F$-algebras when $F$ is perfect. Over a nonperfect field $F$, the separable $F$-algebras are exactly those finite dimensional semisimple $F$-algebras whose each simple factor is a matrix algebra over a division algebra with center which is a separable extension of $F$. In particular, group algebras $F G$, where $G$ is a finite group and $F$ is a field such that the order of $G$ is invertible in $F$, are separable.

The main result of this paper, Theorem 1, states that every separable algebra over an infinite field $F$ admits a presentation with 2 generators and finitely many relations. A different proof of a special case of this result, when the algebra is a direct sum of matrix algebras over $F$, has been given in $[8$, Theorem $3.11]$ for $F=\mathbb{Q}$ and in $[6$, Theorem A1] for an arbitrary infinite field $F$. Theorem 1 is no longer true when $F$ is a finite field. A detailed study of generating sets of finite dimensional separable algebras over finite fields and some algebras over rings of algebraic integers has been initiated in [6] and then extended and generalized in [7].

It is our conviction that presentations of algebras are not only interesting in their own right but also because they have applications to the algebras they describe. In the last section we show how to use presentations to find a polynomial criterion to decide when 2 ordered pairs of $2 \times 2$ matrices $(A, B)$ and $\left(A^{\prime}, B^{\prime}\right)$ with entries in a commutative ring $R$ are automorphically conjugate over the matrix algebra $M_{2}(R)$, under an additional assumption that both pairs generate $M_{2}(R)$ as an $R$-algebra. This generalizes a classical result in invariant theory [4]. Some related ideas have been recently studied in [3].

The goals of further research stemming from this paper should be to find simple presentations of concrete separable algebras and to study their presentation ideals. Furthermore, it is desirable to extend Theorem 2 below to both higher dimensions and triples, quadruples, etc. of generating matrices.

2. Proof of the main result. All rings and algebras are assumed throughout this paper to be associative and unital. For a commutative ring $R$, we denote by $R\left\{x_{1}, \ldots, x_{n}\right\}$ the free associative $R$-algebra in $x_{1}, \ldots, x_{n}$. We will often call the elements of $R\left\{x_{1}, \ldots, x_{n}\right\}$ noncommutative polynomials.

We start with some simple observations.

Proposition 1. Let $R$ be a commutative Noetherian ring, and let $A$ be an $R$-algebra which is finitely generated as an $R$-module. Then the kernel of any homomorphism from the free associative algebra $R\left\{x_{1}, \ldots, x_{n}\right\}$ to $A$ is a finitely generated ideal.

Proof. Put $U=R\left\{x_{1}, \ldots, x_{n}\right\}$ and let $f: U \longrightarrow A$ be a homomorphism of $R$-algebras. Set $a_{i}=f\left(x_{i}\right)$ for $i=1, \ldots, n$. Since $A$ is a Noetherian $R$-module 
and the image of $f$ is a submodule of $A$ generated by all noncommutative monomials in $a_{1}, \ldots, a_{n}$, a finite subset of these monomials generates $f(A)$ as an $R$-module. It follows that there is $k$ such that every noncommutative monomial in $a_{1}, \ldots, a_{n}$ of degree $k$ is an $R$-linear combination of monomials of degree $<k$. Therefore, for each monomial $M$ in $x_{1}, \ldots, x_{n}$ of degree $k$ there is a polynomial $g_{M} \in U$ of degree $<k$ such that $M-g_{M} \in \operatorname{ker} f$. Let $J$ be the ideal of $U$ generated by all the polynomials $M-g_{M}$. The $R$-module $U / J$ is finitely generated because it is generated by all the monomials in $a_{1}, \ldots, a_{n}$ of degree $<k$. Since $R$ is Noetherian, the submodule $\operatorname{ker} f / J$ of $U / J$ is also a finitely generated $R$-module. Thus ker $f / J$ is finitely generated as a left $U$-module. Since the ideal $J$ is finitely generated, so is $\operatorname{ker} f$.

The lower bound in the following lemma is not optimal in many particular cases (for example, for the algebra of $2 \times 2$ matrices over any field it is 2 instead of 4 ), but the result is sufficient for our purposes.

Lemma 1. Let $A$ be an $m$-dimensional algebra over a field $F$. Elements $a_{1}, \ldots$, $a_{k}$ generate $A$ as an $F$-algebra if and only if all the noncommutative monomials of degree $\leq m$ in $a_{1}, \ldots, a_{k}$ span $A$ as a vector space over $F$.

Proof. Let $A_{i}$ be the subspace of $A$ spanned by all the monomials in $a_{1}, \ldots, a_{k}$ of degree $\leq i$. Clearly $A_{1} \subseteq A_{2} \subseteq A_{3} \subseteq \cdots$. We also see that

$$
A_{i+1}=A_{i}+a_{1} A_{i}+a_{2} A_{i}+\cdots+a_{m} A_{i}
$$

for any $i$. It follows that if $A_{i}=A_{i+1}$ for some $i$, then $A_{j}=A_{i}$ for all $j \geq i$.

If $A_{m} \neq A$ then $\operatorname{dim}_{F} A_{m}<m$, so $A_{i}=A_{i+1}$ for some $i<m$. It follows that $A_{i}=A_{m}$ for all $i \geq m$ and therefore $A_{m}$ is a proper subalgebra of $A$. In other words, $a_{1}, \ldots, a_{k} \in A$ do not generate $A$ as an $F$-algebra. This proves that if $a_{1}, \ldots, a_{k} \in A$ generate $A$ as an $F$-algebra then $A=A_{m}$. The converse implication is clear.

Proposition 2. Let $A$ be a finite dimensional algebra over an infinite field $F$. Let $L$ be a field extension of $F$. Then $A$ can be generated as an $F$-algebra by $k$ elements if and only if $A \otimes_{F} L$ can be generated as an L-algebra by $k$ elements. The set

$$
G_{F}(A, k)=\left\{\left(v_{1}, \ldots, v_{k}\right) \in A^{k}: v_{1}, \ldots, v_{k} \text { generate the } F \text {-algebra } A\right\}
$$

is open in the Zariski topology on $A^{k}$ obtained by considering $A^{k}$ as an F-vector space.

Proof. In what follows, we consider $A$ as a subset of $A \otimes_{F} L$ by identifying every $a \in A$ with $a \otimes 1 \in A \otimes_{F} L$. Let $u_{1}, \ldots, u_{m}$ be a basis of $A$ over $F$. Every element $a$ of $A$ (respectively of $A \otimes_{F} L$ ) can be expressed in a unique way as $a=$ $\sum_{i=1}^{m} t_{i} u_{i}$ for some $t_{i} \in F$ (respectively for some $t_{i} \in L$ ). Consider $k$ elements $a_{1}, \ldots, a_{k}$ in $A$ (respectively in $A \otimes_{F} L$ ). Write $a_{j}=\sum_{i=1}^{m} a_{i, j} u_{i}$ for $j=1, \ldots, k$ and for some $a_{i, j} \in F$ (respectively for some $a_{i, j} \in L$ ). By Lemma 1, the elements $a_{1}, \ldots, a_{k}$ generate $A$ (respectively $A \otimes_{F} L$ ) if and only if the monomials of degree $\leq m$ in $a_{1}, \ldots, a_{k}$ span $A$ (respectively $A \otimes_{F} L$ ) as a vector space over $F$ (respectively over $L$ ). Consider a monomial $M=M\left(x_{1}, \ldots, x_{k}\right)$ in $k$ 
variables. There are polynomials $p_{j}^{M}\left(x_{1,1}, \ldots, x_{m, k}\right) \in F\left[x_{1,1}, \ldots, x_{m, k}\right]$ such that $M\left(a_{1}, \ldots, a_{k}\right)=\sum_{i=1}^{m} p_{i}^{M}\left(a_{1,1}, \ldots, a_{m, k}\right) u_{i}$ for any $a_{1}, \ldots, a_{k} \in A \otimes_{F} L$.

Let $B$ be the matrix whose rows are labeled in some way by the monomials $M$ in $x_{1}, \ldots, x_{k}$ of degree $\leq m$, and whose row with label $M$ is

$$
\left(p_{1}^{M}\left(x_{1,1}, \ldots, x_{m, k}\right), \ldots, p_{m}^{M}\left(x_{1,1}, \ldots, x_{m, k}\right)\right) .
$$

The $m \times m$ minors of $B$ are polynomials in $F\left[x_{1,1}, \ldots, x_{m, k}\right]$ and the elements $a_{1}, \ldots, a_{k}$ do not generate $A$ (respectively $A \otimes_{F} L$ ) as an $F$-algebra (respectively as an $L$-algebra) if and only if all these polynomials evaluated at $x_{i, j}=a_{i, j}$ vanish. Therefore, $A$ cannot be generated by $k$ elements as an $F$-algebra if and only if all the $m \times m$ minors of $B$ vanish for every choice of $x_{i, j} \in F$. Since $F$ is infinite, this means that each $m \times m$ minor of $B$ is the zero polynomial. Therefore, each of these minors vanishes for every choice of $x_{i, j} \in L$, and consequently $A \otimes_{F} L$ cannot be generated by $k$ elements as an $L$-algebra. This proves that if $A \otimes_{F} L$ can be generated by $k$ elements as an $L$-algebra, then $A$ can be generated by $k$ elements as an $F$-algebra.

Conversely, if $A$ can be generated by $k$ elements as an $F$-algebra, then clearly the same elements generate $A \otimes_{F} L$ as an $L$-algebra.

Finally, a choice of a basis of $A$ over $F$ allows us to identify $A^{k}$ with $F^{k n}$ and therefore to define the Zariski topology on $A^{k}$ (which does not depend on the choice of a basis). We have proved above that the complement of the set $\mathrm{G}_{F}(A, k)$, defined in (1), is a closed subset of $A^{k}$ consisting of common zeros of all the $m \times m$ minors of the matrix $B$. Thus $\mathrm{G}_{F}(A, k)$ is open in the Zariski topology.

As an immediate corollary we obtain the well known Primitive Element Theorem from commutative algebra.

Corollary 1. Let $A$ be a separable commutative algebra over an infinite field $F$. Then $A$ is a cyclic F-algebra.

Proof. Let $\bar{F}$ be an algebraic closure of the field $F$. It suffices to prove that $A \otimes_{F} \bar{F}$ is generated by one element as an $\bar{F}$-algebra. Since $A$ is separable, it follows from Definition 1 that $A \otimes_{F} \bar{F}=\bar{F}^{m}$ for some $m$. Let $a_{1}, \ldots, a_{m}$ be pairwise distinct elements of $\bar{F}$. Then the Chinese Remainder Theorem implies that the $\bar{F}$-algebras $\bar{F}^{m}$ and $\bar{F}[x] /\left(\left(x-a_{1}\right) \ldots\left(x-a_{m}\right)\right)$ are isomorphic. Hence $A \otimes_{F} \bar{F}$ is generated by a single element as an $\bar{F}$-algebra.

Let $E_{i j}$ denote the matrix whose $(i, j)$ entry is 1 and all other entries are 0 . The following result is well known, see for example [8, Theorem 3.1].

Lemma 2. Let $R$ be a commutative ring. For any $n \geq 1$ the matrices $E_{11}$ and $E_{1 n}+\sum_{i=1}^{n-1} E_{i+1, i}$ generate $M_{n}(R)$ as an $R$-algebra.

For our proof of Theorem 1 we need a stronger version of Lemma 2 when $R$ is an infinite field, which is stated in the next proposition.

Proposition 3. Let $F$ be an infinite field and let $n \geq 1$. There are matrices $A, B \in M_{n}(F)$ generating $M_{n}(F)$ as an F-algebra and such that the 
$F$-algebras $F[A]$ and $F[B]$ are separable. The set of such pairs of matrices is open in the Zariski topology on $M_{n}(F)^{2}$.

Proof. Choose an $F$-basis of $M_{n}(F)$. It allows us to identify $M_{n}(F)$ with $F^{n^{2}}$ and $M_{n}(F)^{2}$ with $F^{2 n^{2}}$. These identifications induce the Zariski topology on $M_{n}(F)$ and $M_{n}(F)^{2}$ respectively. By Proposition 2, the set $G_{n}(F)$ of all $(A, B) \in M_{n}(F)^{2}$ which generate $M_{n}(F)$ as an $F$-algebra is open in the Zariski topology. By Lemma 2, this set is nonempty. The set $\mathcal{A}$ of all matrices $A \in M_{n}(F)$ which have $n$ different eigenvalues is nonempty. It is open in the Zariski topology because it is given by the condition that the resultant of the characteristic polynomial of $A$ with its first derivative be nonzero. The set $\mathcal{B}$ of $(A, B) \in M_{n}(F)^{2}$ such that both $A$ and $B$ have $n$ different eigenvalues is nonempty. It is open in the Zariski topology because $\mathcal{B}=\mathcal{A}^{2}$. Therefore, $G_{n}(F) \cap \mathcal{B}$ is a nonempty open set in the Zariski topology. It remains to note that if $A$ has $n$ distinct eigenvalues, then the algebra $F[A]$ is separable over $F$.

Now we state and prove the main result of this paper.

Theorem 1. Let $F$ be an infinite field and let $A$ be a finite dimensional, separable $F$-algebra. Then $A$ has a presentation with 2 generators and finitely many relations.

Proof. First we prove that $A$ has 2 generators as an $F$-algebra. By Proposition 2, it suffices to prove that $A \otimes_{F} L$ is generated by two elements as and $L$-algebra, where $L$ is an algebraic closure of $F$. Now $A \otimes_{F} L$ is a finite direct sum $\oplus_{i} B_{i}$ of matrix algebras over $L$. For each $i$, there exist elements $a_{i}, b_{i} \in B_{i}$ which generate $B_{i}$ as an $L$-algebra and such that $L\left[a_{i}\right], L\left[b_{i}\right]$ are separable $L$-algebras. Let $T=\oplus_{i} L\left[a_{i}\right], S=\oplus_{i} L\left[b_{i}\right]$. Thus both $T$ and $S$ are commutative, separable $L$-algebras and therefore $T=F[a], S=F[b]$ for some $a, b$ by Corollary 1 . It follows that $a, b$ generate $A \otimes_{F} L$ as an $L$-algebra.

Since the algebra $A$ admits 2 generators, any such pair of generators defines a finite presentation of $A$ by Proposition 1 with $n=2$.

Remark 1. Theorem 1 is false for any finite field $F$. In fact, since $M_{n}(F)$ is finite, it follows from the pigeonhole principle that for a given $k>0$, if $m$ is sufficiently large then for any $k$ elements of $M_{n}(F)^{m}$ there are two factors of the product $M_{n}(F)^{m}$ such that each of the $k$ elements has the same component in each of the two factors. It follows that for a sufficiently large $m$ the algebra $M_{n}(F)^{m}$ cannot be generated by $k$ elements. Some formulas for the smallest number of generators of such algebras have been obtained in [6] and [7].

Also, the assumption about separability in Theorem 1 is essential as the following example shows. Let $V$ be a $d$-dimensional vector space over a field $L$ and let $A=L \oplus V$ be the algebra with componentwise addition, and multiplication given by $(a, v)(b, w)=(a b, a w+b v)$. Then $A$ cannot be generated by less than $d$ elements as an $L$-algebra. 
3. A conjugacy criterion for 2 pairs of $2 \times 2$ matrices over a commutative ring. A classical topic in invariant theory and representation theory is to classify pairs of matrices under simultaneous conjugation. It is a hard problem in high dimensions but for $2 \times 2$ matrices over $\mathbb{C}$ it has been worked out by Kraft in [4]. To discuss it in more details we introduce the following notation. For a commutative ring $R$ we define as in [8, p. 16] the set

$$
G_{2}(R)=\left\{(A, B) \in M_{2}(R)^{2}: A, B \text { generate } M_{2}(R) \text { as an } R \text {-algebra }\right\} .
$$

The set $G_{2}(R)$ is nonempty by Lemma 2. By [6, Theorem 2.5], the set $G_{2}(R)$ can be described as follows: ${ }^{2}$

$$
G_{2}(R)=\left\{(A, B) \in M_{2}(R)^{2}: \operatorname{det}[A, B] \text { is invertible in } R\right\} .
$$

The group $P G L_{2}(R)$ acts on this set by simultaneous conjugation. In [4] it was proved that two elements $(A, B)$ and $\left(A^{\prime}, B^{\prime}\right)$ of $G_{2}(\mathbb{C})$ belong to the same orbit if and only if

$$
\begin{aligned}
& \operatorname{det} A=\operatorname{det} A^{\prime}, \quad \operatorname{det} B=\operatorname{det} B^{\prime}, \quad \operatorname{tr} A=\operatorname{tr} A^{\prime}, \quad \operatorname{tr} B=\operatorname{tr} B^{\prime}, \\
& \operatorname{tr}(A B)=\operatorname{tr}\left(A^{\prime} B^{\prime}\right) .
\end{aligned}
$$

We will illustrate the usefulness of algebra presentations by using them to give a new simplified proof of the above criterion, which works over arbitrary commutative rings. We define the map conj : $M_{2}(R)^{2} \rightarrow R^{5}$ by

$$
\operatorname{conj}(A, B)=(\operatorname{tr}(A), \operatorname{det}(A), \operatorname{tr}(B), \operatorname{det}(B), \operatorname{det}(A+B)) .
$$

The second author is grateful to Hendrik W. Lenstra for generously sharing his ideas some of which have been used in the following theorem.

Theorem 2. Two pairs $(A, B),\left(A^{\prime}, B^{\prime}\right) \in G_{2}(R)$ satisfy $\operatorname{conj}(A, B)=$ $\operatorname{conj}\left(A^{\prime}, B^{\prime}\right)$ if and only if there is an R-algebra automorphism $f$ of $M_{2}(R)$ such that $f(A)=A^{\prime}$ and $f(B)=B^{\prime}$. If, in addition, every rank one projective $R$-module is free, then there exists a matrix $C \in G L_{2}(R)$ such that $A^{\prime}=C^{-1} A C$ and $B^{\prime}=C^{-1} B C$ if and only if $\operatorname{conj}(A, B)=\operatorname{conj}\left(A^{\prime}, B^{\prime}\right)$.

The above mentioned result of Kraft from [4] is a special case of Theorem 2 by the Skolem-Noether Theorem. Note that (4) compares in the 5th component traces of products, while the 5 th component of (5) is the determinant of the sum. Nevertheless, these two quintuples of invariants are equivalent because of the following identity of $2 \times 2$ matrices over any commutative ring:

$$
\operatorname{tr}(A) \operatorname{tr}(B)-\operatorname{tr}(A B)+\operatorname{det}(A)+\operatorname{det}(B)-\operatorname{det}(A+B)=0 .
$$

We remark that Theorem 2 is false if we do not require that the matrix pairs generate the matrix algebra. For example, $\operatorname{conj}(0,0)=\operatorname{conj}\left(0, E_{12}\right)=$ $(0,0,0,0,0)$ yet the pairs $(0,0)$ and $\left(0, E_{12}\right)$ are not conjugate (recall that $E_{i j}$ is the matrix whose $(i, j)$ entry is 1 and all other entries are 0$)$.

Theorem 2 is proved at the end of this section after some preliminary observations.

Lemma 3. Let $R$ be a commutative ring and let $f: M_{2}(R) \longrightarrow M_{2}(R)$ be an $R$-algebra isomorphism. Then $f$ preserves trace and determinant.

${ }^{2}$ As usual, if $a, b$ are elements of some algebra, then we put $[a, b]=a b-b a$. 
Proof. The only monic polynomial of degree $\leq 2$ over $R$ annihilating $E_{11}$ or $E_{22}$ is $x^{2}-x$. Similarly, the only monic polynomial of degree $\leq 2$ over $R$ annihilating $E_{12}$ or $E_{21}$ is $x^{2}$. Thus the same is true for the images of $E_{11}$, $E_{22}, E_{12}, E_{21}$ under $f$. By the Cayley-Hamilton Theorem, a $2 \times 2$ matrix $U$ satisfies the identity $U^{2}-(\operatorname{tr} U) U+\operatorname{det} U=0$. It follows that the map $f$ preserves traces and determinants of $E_{11}, E_{22}, E_{12}, E_{21}$. Any $X \in M_{2}(R)$ can be written as $a E_{11}+b E_{12}+c E_{21}+d E_{22}$ for some $a, b, c, d \in R$. Applying the map tro $f$ to this expression for $X$, we see that $\operatorname{tr}(f(X))=a+d=\operatorname{tr} X$. Therefore, $f$ preserves the trace. Finally, since $\operatorname{det}(X) I=(\operatorname{tr} X) X-X^{2}$, it follows that $f$ also preserves the determinant.

Consider now the free associative $R$-algebra $R\{x, y\}$ in 2 variables. For $t_{x}, d_{x}$, $t_{y}, d_{y}, d \in R$ consider the ideal

$$
\begin{aligned}
& I\left(t_{x}, d_{x}, t_{y}, d_{y}, d\right)= \\
& \quad\left\langle x^{2}-t_{x} x+d_{x}, y^{2}-t_{y} y+d_{y}, x y+y x-\left(t_{x} y+t_{y} x+d_{x}+d_{y}-d\right)\right\rangle .
\end{aligned}
$$

For matrices $X, Y \in M_{2}(R)$ consider the substitution homomorphism

$$
\eta: R\{x, y\} \longrightarrow M_{2}(R), \quad \eta(x)=X, \quad \eta(y)=Y .
$$

Let $t_{x}, d_{x}, t_{y}, d_{y}, d$ be $\operatorname{tr}(X), \operatorname{det}(X), \operatorname{tr}(Y), \operatorname{det}(Y), \operatorname{det}(X+Y)$, respectively; they are the coordinate maps of conj defined by (5). Let

$$
I(X, Y)=\operatorname{ker} \eta \text {. }
$$

We have the following result relating (6) and (8).

Lemma 4. The inclusion $I\left(t_{x}, d_{x}, t_{y}, d_{y}, d\right) \subseteq I(X, Y)$ holds, and it is an equality when $\eta$ is onto.

Proof. The first claim of the lemma follows from the Cayley-Hamilton Theorem. For the second claim observe that the $R$-module $M=R\{x, y\} / I\left(t_{x}, d_{x}, t_{y}\right.$, $\left.d_{y}, d\right)$ is generated by the images of $1, x, y, x y$ under the quotient map $R\{x, y\} \longrightarrow M$. Thus there exists an $R$-module epimorphism $\gamma: R^{4} \longrightarrow M$. Let $\tilde{\eta}: M \longrightarrow M_{2}(R)$ be the map induced by $\eta$, and let $\delta: M_{2}(R) \longrightarrow R^{4}$ be the map $\left(\begin{array}{ll}a & b \\ c & d\end{array}\right) \mapsto(a, b, c, d)$. If $\eta$ is surjective, then so is $\tilde{\eta}$, and therefore so is $\epsilon=\delta \circ \tilde{\eta} \circ \gamma: R^{4} \longrightarrow R^{4}$. Since $\epsilon$ is a homomorphism of a finitely generated $R$-module onto itself, it follows that $\epsilon$ is an $R$-module automorphism. Thus $\tilde{\eta}$ is an $R$-module isomorphism and therefore $I\left(t_{x}, d_{x}, t_{y}, d_{y}, d\right)=I(X, Y)$.

Lemma 5. Let $\eta_{1}, \eta_{2}$ be R-algebra epimorphisms $R\{x, y\} \longrightarrow M_{2}(R)$. Let $X_{i}=$ $\eta_{i}(x), Y_{i}=\eta_{i}(y)$ for $i=1,2$. Then $I\left(X_{1}, Y_{1}\right)=I\left(X_{2}, Y_{2}\right)$ if and only if there exists an automorphism $\beta$ of $M_{2}(R)$ such that $\beta\left(X_{1}\right)=X_{2}$ and $\beta\left(Y_{1}\right)=Y_{2}$.

Proof. If the automorphism $\beta$ exists then clearly $\beta \circ \eta_{1}=\eta_{2}$ and therefore $I\left(X_{1}, Y_{1}\right)=I\left(X_{2}, Y_{2}\right)$. Conversely, if $I\left(X_{1}, Y_{1}\right)=I\left(X_{2}, Y_{2}\right)=I$ then $\eta_{i}$ induces an isomorphism $\bar{\eta}_{i}: R\{x, y\} / I\left(X_{i}, Y_{i}\right) \longrightarrow M_{2}(R), i=1,2$, and $\beta=\bar{\eta}_{2} \circ \bar{\eta}_{1}^{-1}$ works. 
We can strengthen the above result as follows.

Proposition 4. Let $\eta_{1}, \eta_{2}$ be $R$-algebra epimorphisms $R\{x, y\} \longrightarrow M_{2}(R)$. Let $X_{i}=\eta_{i}(x), Y_{i}=\eta_{i}(y)$ for $i=1,2$. Then $I\left(X_{1}, Y_{1}\right)=I\left(X_{2}, Y_{2}\right)$ if and only if $\operatorname{conj}\left(X_{1}, Y_{1}\right)=\operatorname{conj}\left(X_{2}, Y_{2}\right)$.

Proof. If the invariants are the same, then the kernels coincide by Lemma 4 . If the kernels coincide, then there is an automorphism $f: M_{2}(R) \longrightarrow M_{2}(R)$ such that $f \circ \eta_{1}=\eta_{2}$ by Lemma 5 . Thus the five invariants coincide by Lemma 3 .

Proof of Theorem 2. Let $(A, B),\left(A^{\prime}, B^{\prime}\right) \in G_{2}(R)$. There are $R$-algebra epimorphisms $\eta_{1}, \eta_{2}: R\{x, y\} \longrightarrow M_{2}(R)$ such that $\eta_{1}(x)=A, \eta_{1}(y)=B$, $\eta_{2}(x)=A^{\prime}, \eta_{2}(y)=B^{\prime}$. By Proposition 4 , we have $\operatorname{conj}(A, B)=\operatorname{conj}\left(A^{\prime}, B^{\prime}\right)$ if and only if $\operatorname{ker} \eta_{1}=\operatorname{ker} \eta_{2}$, which by Lemma 5 is equivalent to the existence of the automorphism $f$.

If in addition every rank one projective $R$-module is free then our claim follows from the theorem of Auslander and Goldman [2, Theorem 3.6] stating that over such ring $R$ every automorphism of a separable central $R$-algebra is inner.

\section{References}

[1] G. Agnarsson, S. A. Amitsur, and J. C. Robson, Recognition of matrix rings. II, Israel J. Math. 96 (1996), 1-13.

[2] M. Auslander And O. Goldman, The Brauer group of a commutative ring, Trans. AMS 97 (1960), 367-409.

[3] A. Fabianska and W. Plesken, An $L_{2}$-quotient algorithm for finitely presented groups, J. Algebra 322 (2009), 914-935.

[4] H. KRAfT, Geometric methods in representation theory, Representations of algebras (Puebla, 1980), pp. 180-258, Lecture Notes in Math., 944, Springer, Berlin-New York, 1982.

[5] K. Kassel, Quantum groups, Graduate Texts in Mathematics 155, SpringerVerlag, New York, 1995.

[6] R. V. Kravchenko and B. V. Petrenko, Some formulas for the smallest number of generators for finite direct sums of matrix algebras, arxiv:math/0611674v7

[7] R. V. Kravehenko, M. Mazur, and B. V. Petrenko, Generators of Algebras, in preparation.

[8] B. V. Petrenko And S. N. Sidki, On pairs of matrices generating matrix rings and their presentations, J. Algebra 310 (2007), 15-40.

[9] R. S. Pierce, Associative Algebras, GTM 88, Springer-Verlag, New York Heidelberg Berlin, 1982.

[10] J. C. Robson, Recognition of matrix rings, Comm. Algebra 19 (1991), 2113-2124. 


\section{MarCin MaZur}

Department of Mathematics,

Binghamton University,

P.O. Box 6000,

Binghamton, NY 13892-6000, USA

e-mail: mazur@math.binghamton.edu

Bogdan V. Petrenko

Department of Mathematics,

SUNY Brockport,

350 New Campus Drive,

Brockport, NY 14420, USA

e-mail: bpetrenk@brockport.edu

Received: 6 August 2009 